Research Paper

\title{
Alcohol accumulation promotes esophagitis via pyroptosis activation
}

Fengjiao Wang ${ }^{*}$, Gang $\mathrm{Li}^{2 *}$, Jinfeng Ning ${ }^{1}$, Lantao Chen ${ }^{1}$, Hai $\mathrm{Xu}^{1}$, Xianglong Kong ${ }^{1}$, Jianlong Bu1, Weiwei Zhao ${ }^{3}$, Zhengtian $\mathrm{Li}^{4}$, Xiuyun Wang ${ }^{5}$, Xiaoguang $\mathrm{Li}^{6}$, Jianqun $\mathrm{Ma}^{1}$

1. Department of Thoracic Surgery, Harbin Medical University Cancer Hospital, Harbin, 150001, China

2. Department of General Surgery, the Second Affiliated Hospital of Harbin Medical University, Harbin, 150000, China

3. Department of Epidemiology and Biostatistics, School of Public Health, Harbin Medical University, Harbin, 150086, China

4. Department of General Surgery, the First Affiliated Hospital of Harbin Medical University, Harbin, 150001, China

5. Department of Abdominal Ultrasound, First Hospital of Harbin Medical University, Harbin, 150001, China

6. Department of Cell Biology, Johns Hopkins University School of Medicine, Baltimore, MD 21205, USA

${ }^{*}$ Authors share co-first authorship.

$\triangle$ Corresponding author: Jianqun Ma, Department of Thoracic Surgery, Harbin Medical University Cancer Hospital, Harbin, 150000, China, Telephone: (86) 15846620781, E-mail: jianqunmahyd@163.com

(C) Ivyspring International Publisher. This is an open access article distributed under the terms of the Creative Commons Attribution (CC BY-NC) license (https://creativecommons.org/licenses/by-nc/4.0/). See http://ivyspring.com/terms for full terms and conditions.

Received: 2017.12.13; Accepted: 2018.06.12; Published: 2018.07.13

\begin{abstract}
Gastroesophageal reflux impairs the mucosal barrier in the distal esophagus, allowing chronic exposure of the squamous epithelium to multitudinous stimulations and inducing chronic inflammation. Esophagitis is a response to inflammation of the esophageal squamous mucosa. Our study clarified that alcohol accumulation could aggravate the progress of esophagitis by inducing pyroptosis; however, Ac-YVAD-CMK, an inhibitor of caspase-1, could effectively suppress the expression of IL-1 $\beta$ and IL-18 both in vivo and in vitro, reducing the inflammatory response, which is promised to be an agent to inhibit the progression of esophagitis. Additionally, caspase-1-derived pyroptosis is involved in esophageal cancer.
\end{abstract}

Key words: alcohol, esophagitis, pyroptosis, caspase-1, Ac-YVAD-CMK

\section{Introduction}

Gastroesophageal reflux disease (GERD) is defined as a disorder in which acid gastric contents reflux recurrently into the esophagus, causing acid regurgitation at least once a week [1-4]. GERD can damage the esophageal mucosa, leading to esophageal bleeding or stricture, and can also trigger long-term complications, including Barrett's esophagus or esophageal adenocarcinoma [5-8]. Previous studies have explicated that the consumption of large amounts of alcohol tends to be associated with an increased risk of erosive esophagitis, promoting acid regurgitation by reducing the pressure of the lower esophageal sphincter and slowing both esophageal motility and gastric emptying, regardless of the type of alcoholic beverage [9-12]. Importantly, acid and pepsin can induce macroscopically detectable injury to the esophageal squamous epithelium. Injured or inflammatory tissues release inflammatory mediators, which can be investigated by the immune system. Moreover, in response to these chemical insults, endothelial cells produce adhesion molecules, which recruit and activate leukocytes, thus mediating inflammatory conditions [13]. Alcohol can affect the esophagus in several ways: it induces mucosal inflammation, increases the risk for Barrett's esophagus and esophageal cancer, and impairs esophageal motility [14-19]. However, the mechanisms involved in the occurrence of esophagitis induced by alcohol remain to be elucidated.

Pyroptosis is an inflammatory cell death and is typically triggered by caspase- 1 after its activation by various inflammasomes [20, 21]. Caspase 1 was initially called interleukin IL-1 $\beta$-converting enzyme 
and was first recognized as a protease that converts the inactive precursors of interleukin $1 \beta$ (IL-1 $1 \beta$ ) and interleukin 18 (IL-18) into mature inflammatory cytokines [22-26]. IL-1 $\beta$ and IL-18 are potent endogenous pyrogens that could stimulate fever, leukocyte tissue migration and expression of diverse cytokines and chemokines [27-30]. Furthermore, IL-1 $\beta$ and IL-18 play crucial roles in the pathogenesis of a range of inflammatory and autoimmune diseases [31]. Caspase- 1 can be activated by a cytoplasmic protein complex called the inflammasome [32], and caspase 1 activation can result in not only the production of activated inflammatory cytokines but also rapid cell death characterized by plasma-membrane rupture, DNA damage and release of proinflammatory intracellular contents [33-36]. Currently, there is no related study showing the association between pyroptosis and the occurrence of esophagitis.

In the present study, we demonstrated that alcohol accumulation could initiate caspase-1 activation and DNA fragmentation and stimulate the activation of inflammatory cytokines IL-1 $\beta$ and IL-18, causing pyroptosis not only in normal esophageal epithelial cell line HEEC but also in vivo. Furthermore, we revealed that pyroptosis was involved in the occurrence of esophagitis, and amplification of the inflammation signal in vivo, accelerating the progression of esophagitis. We also demonstrated the activation of pyroptosis in esophageal cancer.

\section{Results}

Alcohol causes caspase-1 activation in HEECs - The dosage window of alcohol within which it is considered safe was detected using normal human esophageal endothelial cells (HEECs), which were cultured with ethanol diluted by DMEM plus $10 \%$ fetal bovine serum to $0.5 \%, 1 \%, 2 \%, 4 \%, 8 \%$ and $16 \%$, according to the cell survival extent. Finally, the maximal safety use level was confirmed to be $1 \%$ [Figure 1A].

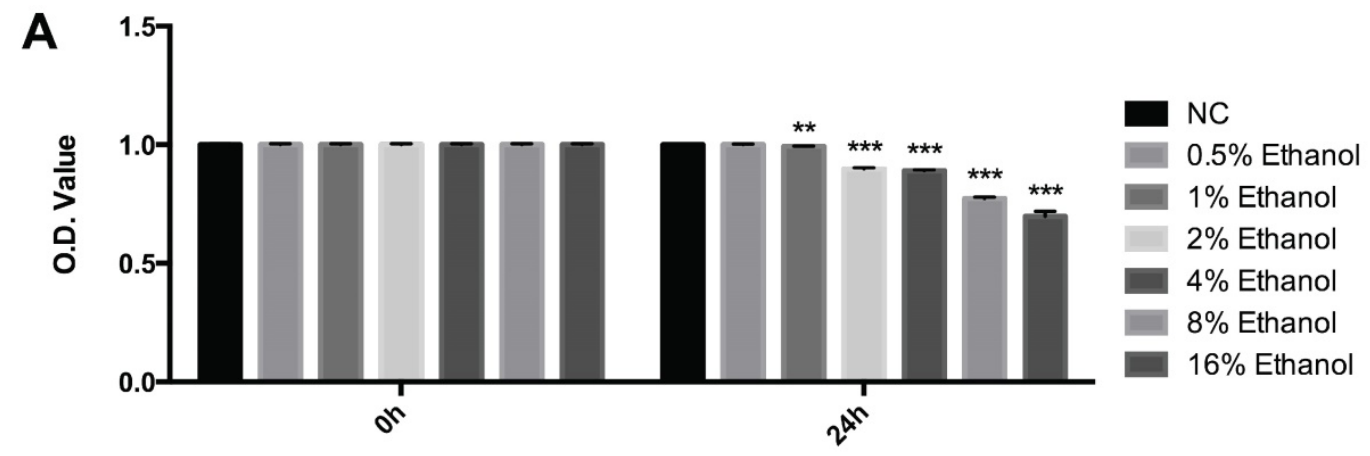

B
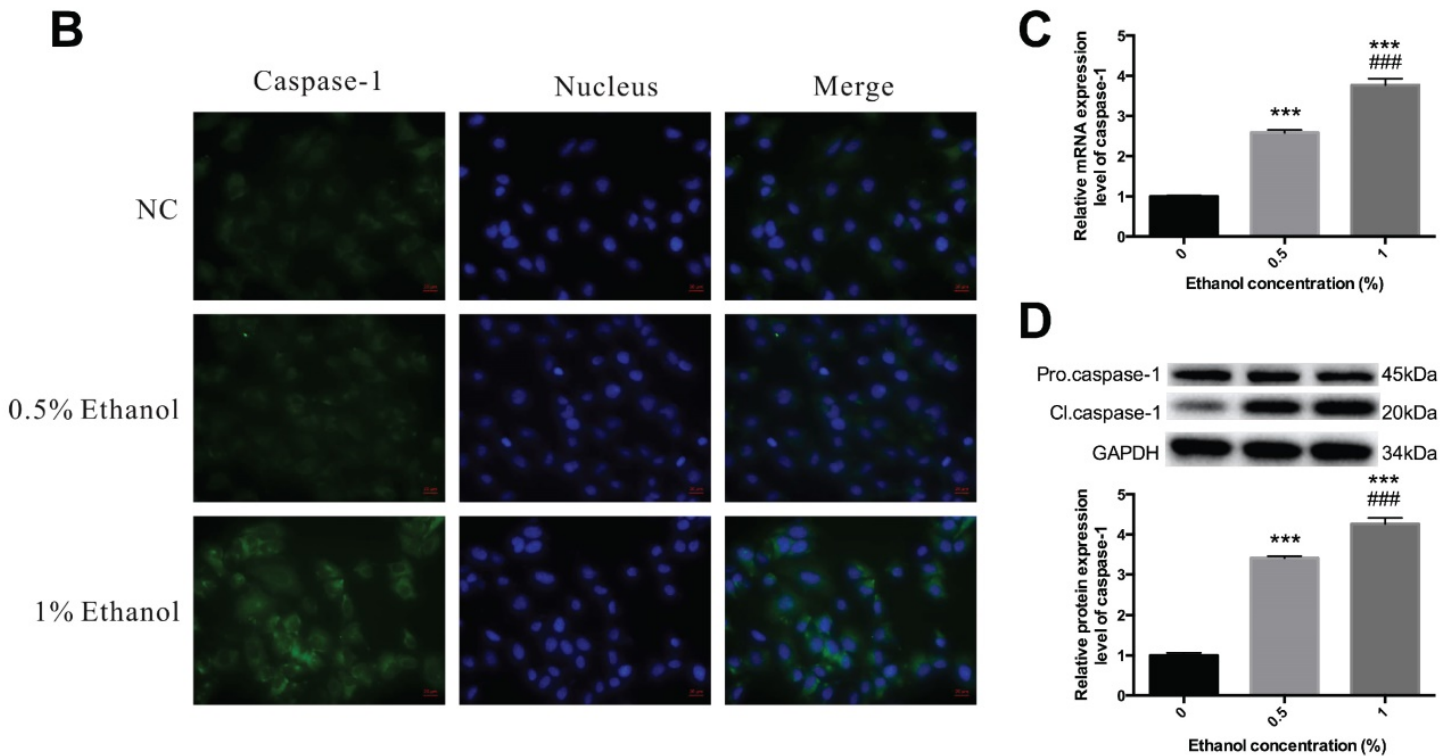

FIGURE 1. Ethanol treatment induces caspase-1 activation. A. HEECs were treated with ethanol at the concentrations of $0,0.5 \%, 1 \%, 2 \%, 4 \%, 8 \%$ and $16 \%$, and the cellular survival rate was detected by the MTT assay. B. HEEC cells were separately treated with $0.5 \%$ ethanol and $1 \%$ ethanol, and caspase- 1 activation, as well as its mRNA and protein expression levels, were detected by immunofluorescence (B), real-time PCR (C) and western blotting (D). The data are representative of five independent experiments and represent the means \pm SD. ${ }^{*}, p<0.01 ; * * *, p<0.001$. \#\#, $p<0.001$. * indicates the comparison between adjacent groups, \# indicates the comparison between the groups independent of each other. 
As an inflammatory process, pyroptosis may play an essential role in the development of esophagitis. To explore whether pyroptosis is involved in esophagitis induced by alcohol, we first detected the expression level of caspase-1. HEECs were treated with ethanol diluted to the concentrations of $0.5 \%$ and $1 \%$, and then we carried out immunofluorescence using caspase- 1 antibody and labeled the nucleus with DAPI. As expected, there was an obvious activation of caspase- 1 at a concentration of 1\% [Figure 1B]. To further confirm that caspase- 1 was activated, we assessed the mRNA and protein expression levels of caspase- 1 by qRT-PCR and western blotting in the HEECs. Thus, total RNA was extracted, and then RT-qPCR was performed, Pro.caspase-1 and Cl.caspase-1 protein were extracted and subjected to Western blot analysis [Figure 1C \& 1D]. The results showed that alcohol treatment could cause caspase- 1 activation.

Alcohol induces cell death in HEECs-Like apoptotic cell death, pyroptosis also shows positive terminal deoxynucleotidyl transferase - mediated dUTP nick end-labeling (TUNEL) staining. Figure 2 shows the fluorescent signals of TUNEL and DAPI staining of HEECs: TUNEL staining monitors DNA damage, and DAPI staining monitors morphological changes in nuclei. In the non-treated group, there was a small number of dead cells. Compared with the non-treated group, the cell death rate was significantly increased at $48 \mathrm{~h}$ after treatment with alcohol in a dose-dependent relationship.

Alcohol activates inflammatory cytokines IL-1 $\beta$ and $I L-18$ - To ensure that it was pyroptosis, not apoptosis, that participated in this process, we further determined whether alcohol could amplify the inflammatory response during esophagitis through pyroptosis. We detected the expression levels of IL-1 $\beta$ and IL-18. HEECs were treated with ethanol diluted to the concentrations of $0.5 \%$ and $1 \%$, and then we performed qPCR and Western blot assays to detect IL-1 $\beta$ and IL-18 expression in different forms [Figure 3]. Compared with the control groups, the mRNA and protein expression levels of mature IL-1 $\beta$ and IL-18 were increased in the alcohol-treated group, further consolidating our results that alcohol could induce pyroptosis and activate the inflammatory response to promote the occurrence and development of esophagitis.

The caspase-1 inhibitor suppresses alcohol-induced pyroptosis and inflammation-Because alcohol treatment could induce pyroptosis, next we detected the effect of the caspase- 1 inhibitor N-Ac-Tyr-Val-AlaAsp-chloromethyl ketone (Ac-YVAD-CMK) on pyroptosis and inflammatory cytokines in esophagitis. We first needed to clarify the inhibitory effect of Ac-YVAD-CMK on caspase-1. Thus, we carried out immunofluorescence assays [Figure 4A]. The results showed that both Ac-YVAD-CMK and caspase-1 siRNA could remarkably inhibit the caspase-1 expression level. Cell death induced by pyroptosis was also suppressed by Ac-YVAD-CMK [Figure 4B]. Furthermore, qRT-PCR and western blotting showed that this agent could effectively inhibit caspase-1 expression similar to siRNA [Figure $4 \mathrm{C} \& 4 \mathrm{D}]$

Next, we detected the effect of the inhibitor on the inflammatory response. We treated HEEC cells with the aforesaid pattern and then detected the mRNA and protein expression levels of different forms of IL-1 $\beta$ and IL-18 [Figure $4 \mathrm{C} \& 4 \mathrm{D}$ ]. The inhibitor-treated group reversed the increased expression of IL-1 $\beta$ and IL-18 compared with the alcohol-treated group. qRT-PCR and western blot assays also showed that the IL-1 $\beta$ and IL-18 mRNA
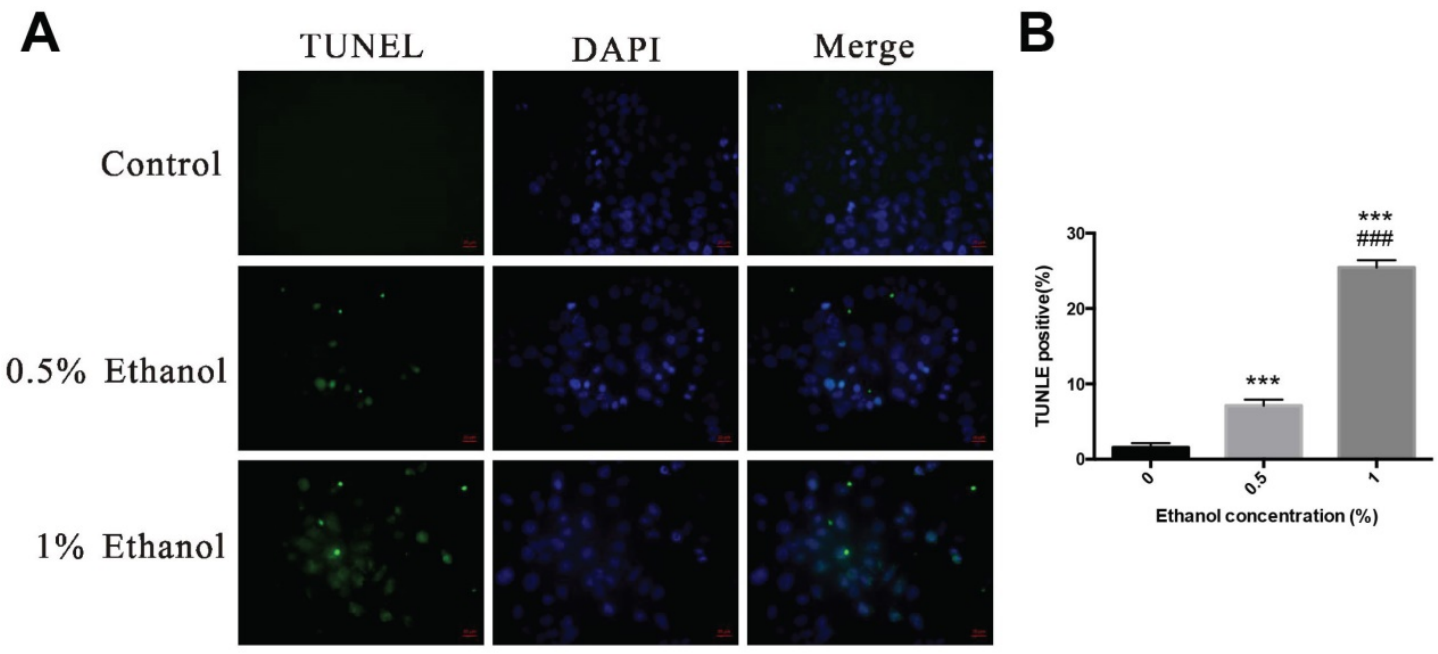

FIGURE 2. Ethanol activates cell death in HEECs. HEECs were treated with $0.5 \%$ ethanol and $1 \%$ ethanol, and cell death was detected by TUNEL.***, $p<0.001$; \#\#\#, $p<0.001$. 
A

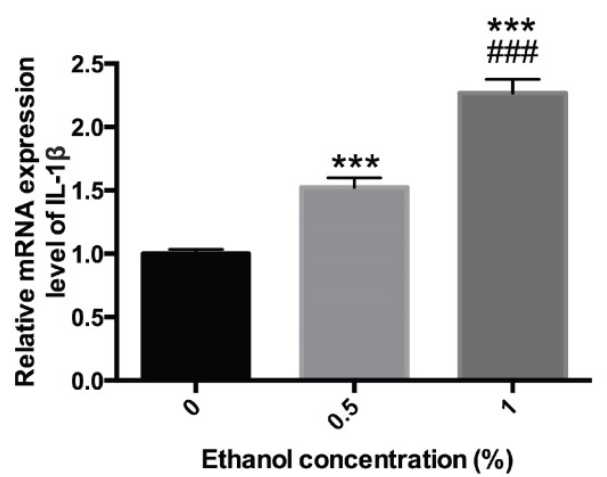

C

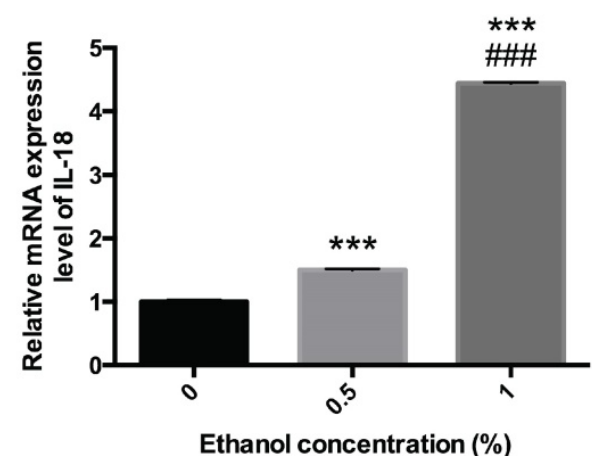

B

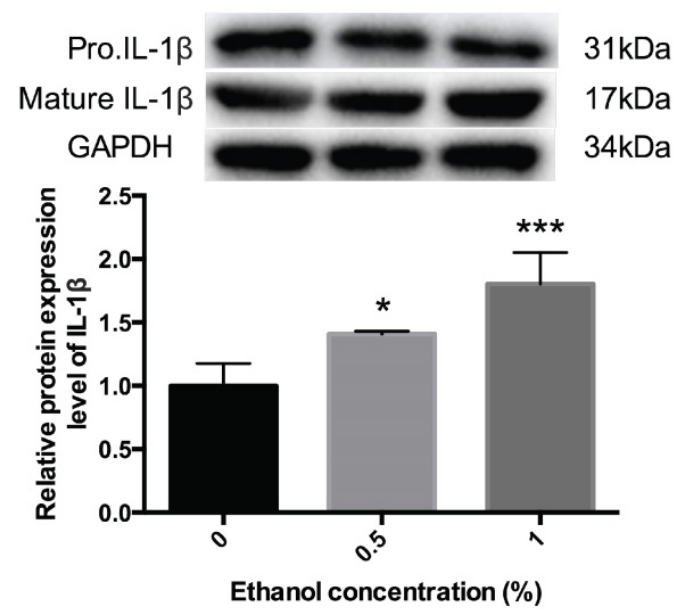

D

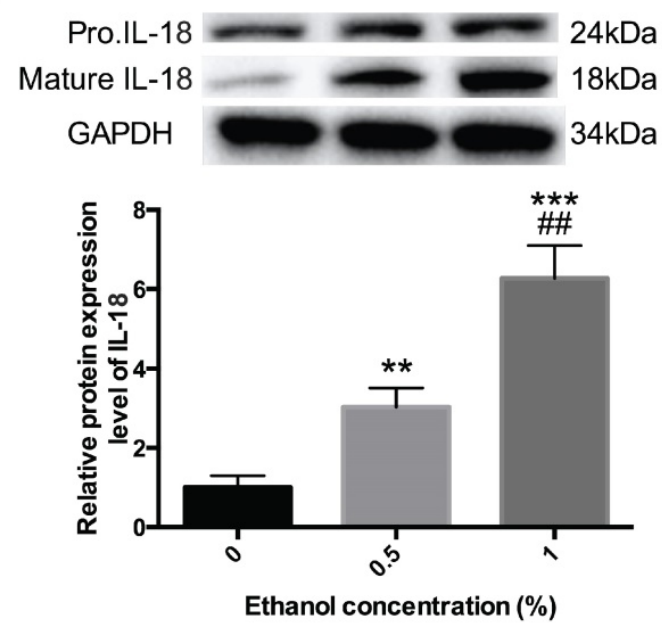

FIGURE 3. IL- $1 \beta$ and IL- 18 were activated in ethanol-treated cells. HEECs were treated with $0.5 \%$ and $1 \%$ ethanol. The activation level of IL- $1 \beta$ and IL- 18 were detected by real-time PCR $(A, C)$ and western blotting $(B, D)$. The data are representative of five independent experiments and represent the means \pm SD. $*, p<0.05$; **, $p<0.01$; ***, $p<0.001$; \#, $p<0.01$; \#\#, $p<0.001$.

and protein expression levels were effectively decreased in the inhibitor-treated group. As expected, the inhibitor could significantly inhibit the expression level of mature IL-1 $\beta$ and IL-18.

Alcohol accumulation initiates pyroptosis in vivo - To further confirm that alcohol drinking could act on esophagitis through pyroptosis, we completed animal experiments. We chose nine Balb/c mice, six to eight weeks old, and divided them into three groups. The first group was given normal saline, the second group was given $1 \%$ alcohol, and the third group was given the same alcohol concentration and the inhibitor Ac-YVAD-CMK. All three groups were administered the agents by gavage. First, we isolated the esophageal epithelial tissues of these three groups to form hematoxylin and eosin (HE) staining [Figure 5A]. Inflammatory cell evasion was detected in these three groups, and the results showed a significant increase in mouse esophageal mucosal inflammation in the ethanol group and then decreased in caspase-1 inhibitor-treated group. Next, we carried out immunohistochemical assays to detect caspase-1, IL-1 $\beta$ and IL-18 expression, and the results showed that alcohol treatment activated the expression of all three; however, the inhibitor significantly reversed the increased expression [Figure 5B-D]. Next, we extracted the esophageal epithelial cells from all three groups, collected the whole nucleic acids and proteins, detected the mRNA [Figure 5E] and protein expression levels [Figure 5F] of caspase-1, IL-1 $\beta$ and IL-18. The results suggested that alcohol drinking could induce caspase-1 activation in esophagitis; however, Ac-YVAD-CMK could inhibit the activation of IL-1 $\beta$ and IL-18 remarkably, reducing the inflammatory response and greatly relieving the progression of esophagitis.

Pyroptosis is activated in esophageal cancer tissues - Because pyroptosis plays an important role in esophagitis induced by alcohol and it has been proved that alcohol has a close relationship with esophageal 
cancer $[14,16]$, we next detected the activation levels of caspase-1, IL-1 $\beta$ and IL-18 in esophageal cancer to identify whether pyroptosis was involved in esophageal cancer progression. We collected 30 paired tissue samples from Harbin Medical University Cancer Hospital, all of whom have a drinking history of more than twenty years. We then detected caspase- 1 [Figure 6A], IL-1 $\beta$ [Figure 6B] and IL-18 [Figure 6C] expression through immunohistochemical staining using esophageal cancer tissues and adjacent normal tissues from 6 random patients. The results showed that the expression levels of both caspase-1, IL-1 $\beta$ and IL-18 were increased in cancer tissues, compared with those in non-tumor tissues. To further identify the expression levels of the three factors in cancerous tissues, we isolated the nucleic acids and proteins from esophageal cancer

A

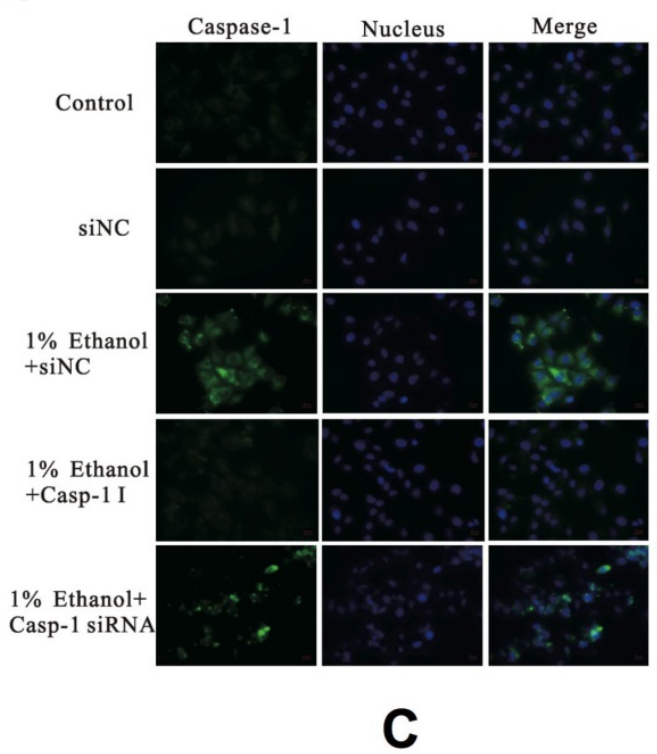

tissues and non-tumor tissues to detect the transcription [Figure 6D] and translation levels [Figure 6E] via qRT-PCR and western blot assays, respectively. The results showed that caspase- 1 , IL-1 $\beta$ and IL-18 were increased in esophageal cancer tissues. These results suggest that pyroptosis may be a main participant in esophageal cancer progression.

\section{Discussion}

In recent years, there has been great progress in studies concerning esophageal cancer; however, very scant reports exist concerning esophagitis. Barrett's esophagus (BE) is considered to develop as a result of chronic gastroesophageal reflux disease (GERD) and to predispose to esophageal adenocarcinoma (EAC) [37], GERD is caused by abnormal reflux of the gastric contents into the

B
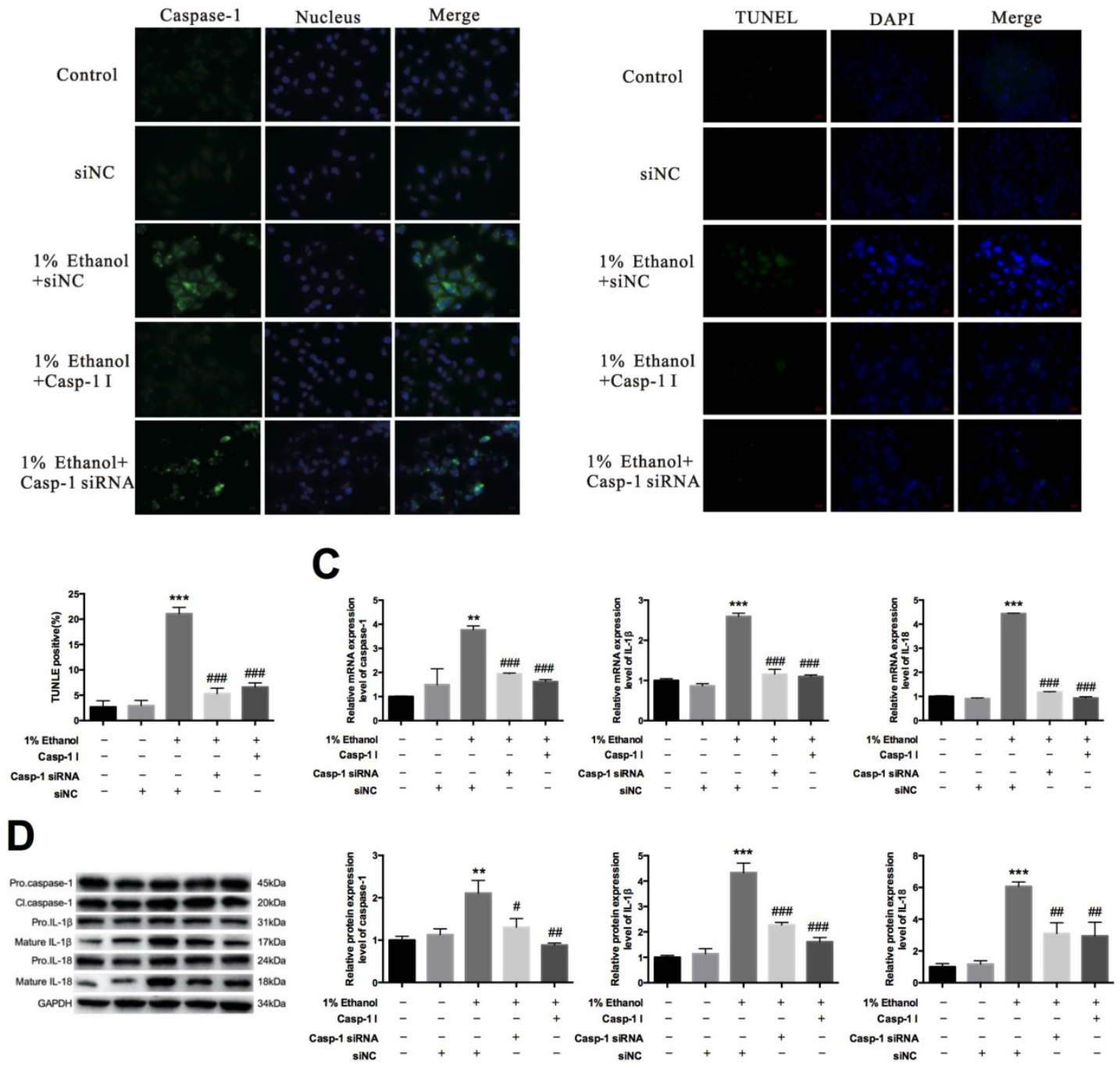

FIGURE 4. Caspase- 1 inhibitor decreases the activation levels of caspase- $1, \mathrm{IL}-1 \beta$ and IL- 18 . HEECs were treated with siNC, $1 \%$ ethanol+siNC, $1 \%$ ethanol+caspase- 1 inhibitor or $1 \%$ ethanol+caspase- 1 siRNA. A. The caspase- 1 activation level was detected by immunofluorescence assay. B. Cell death was detected by TUNEL. The mRNA expression (C) and protein levels in different forms (D) of caspase-1, IL-1 $\beta$ and IL-18 were detected by real-time PCR and western blotting. The data are representative of five independent experiments and represent the means \pm SD. $* *, p<0.01 ; * * *, p<0.001 ; \#, p<0.05 ; \#, p<0.01 ; \ldots \#, p<0.001$. 
esophagus, which is characterized by specific symptoms such as heartburn and acid regurgitation [38-42]. However, only about one-third to one-half of patients with GERD presented endoscopically positive changes, such as erosions and ulcers, while the others with GERD symptoms showed no obvious mucosal damage during endoscopic examination.
A

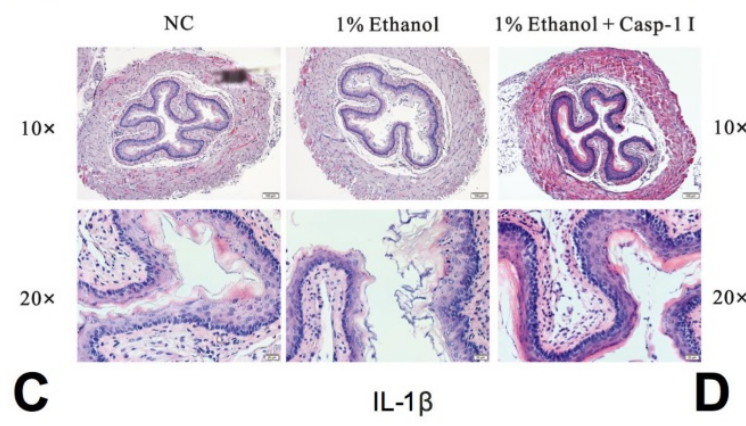

C

IL-1 $\beta$

B
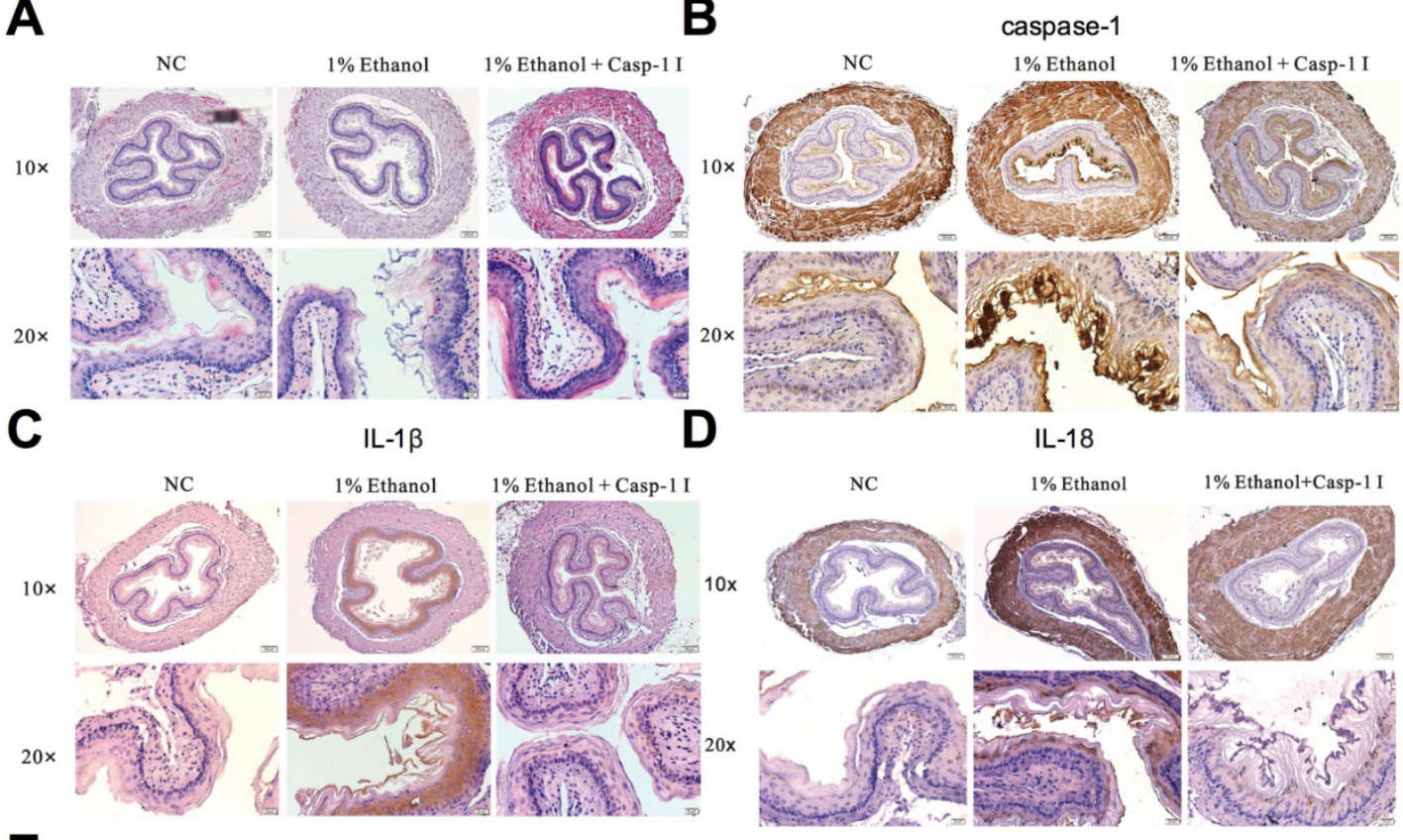

E
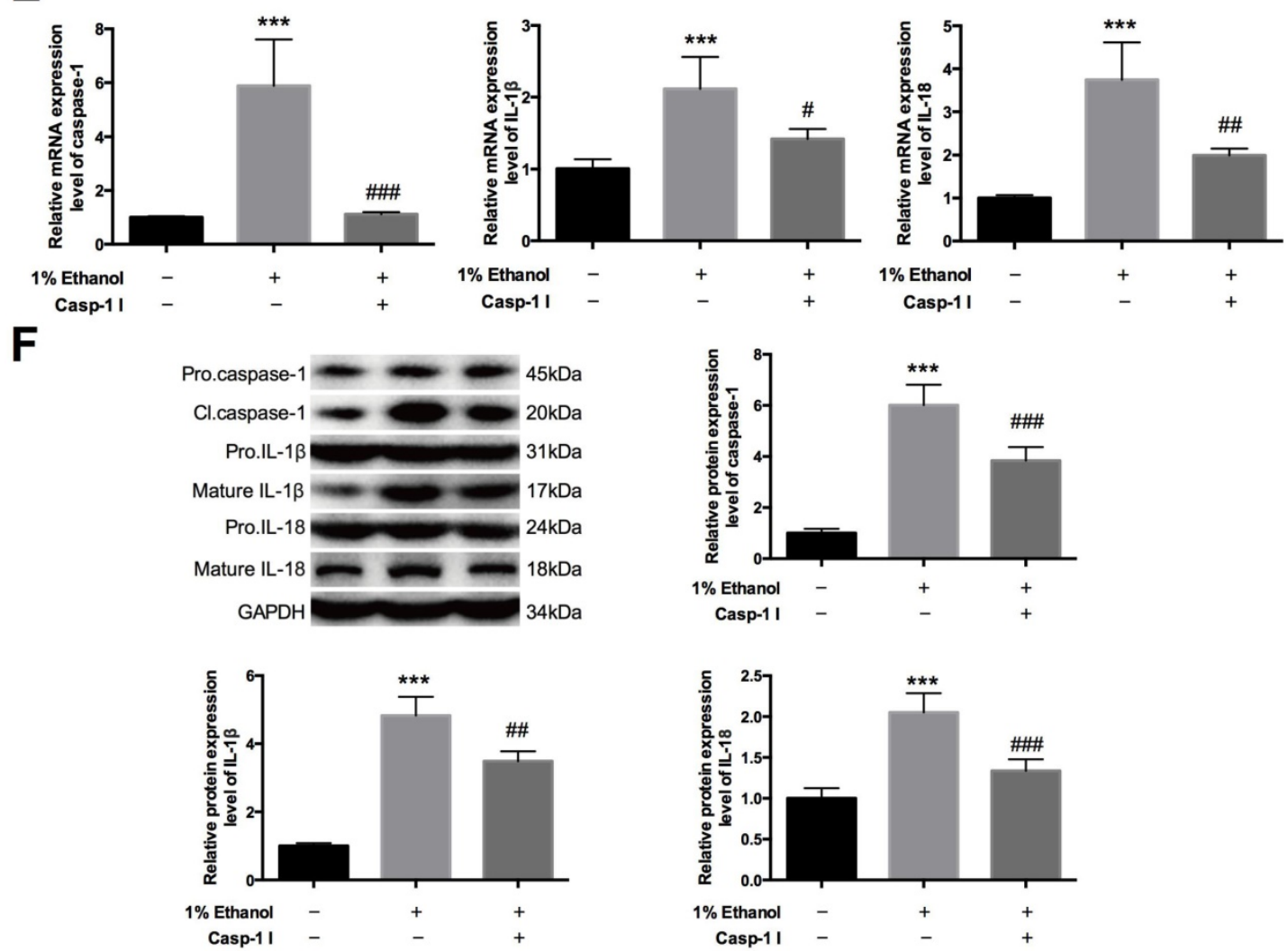

FIGURE 5. Caspase-1 inhibitor decreases the activation of caspase-1, IL-1 $\beta$ and IL-18 induced by ethanol in vivo. The mice were divided into three groups-infused with physiological saline, ethanol and ethanol+caspase-1 inhibitor-with the same volume. Isolation of esophageal tissues and extraction of both nucleic acid and protein. A. Esophageal histopathology of mice was analyzed by hematoxylin and eosin (HE) staining at the indicated times after treatment with ethanol or ethanol and caspase-1 inhibitor treatments (magnification, 10x and 20x). Immunohistochemical staining of caspase-1 (B), IL-1 $\beta$ (C) and IL-18 (D) in the three groups. E. The mRNA expression level of caspase-1, IL-1 $\beta$ and IL-18 was detected by real-time PCR. F. The protein expression level of caspase-1, IL-1 $\beta$ and IL-18 in different forms was detected by western blotting. The data are representative of five independent experiments and represent the means \pm SD. $* * *, p<0.001 ; \#, p<0.05 ; \#, p<0.01 ; \# \#$, $p<0.001$. 


\section{A}

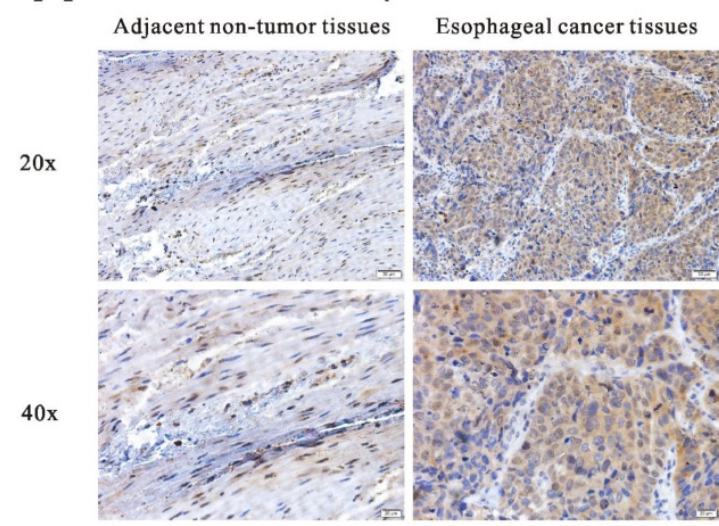

C

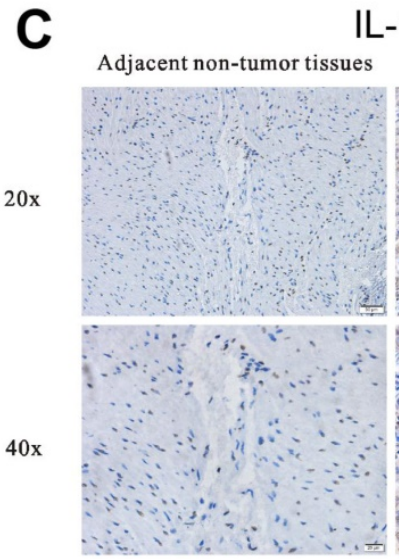

E
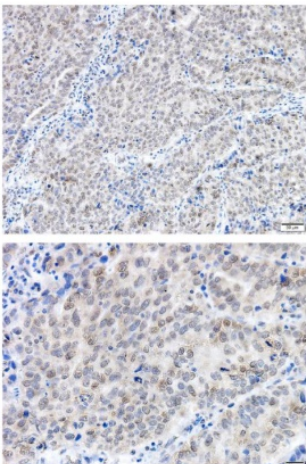
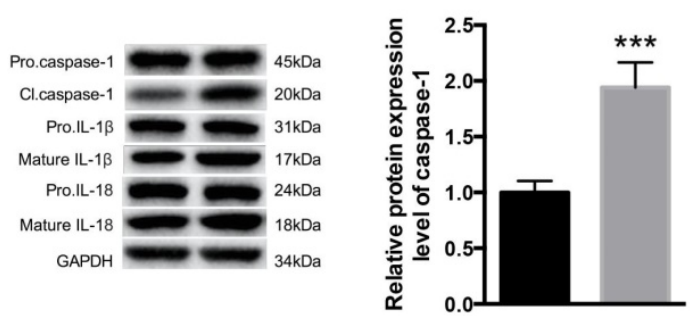

B

$20 \mathrm{x}$

$40 x$

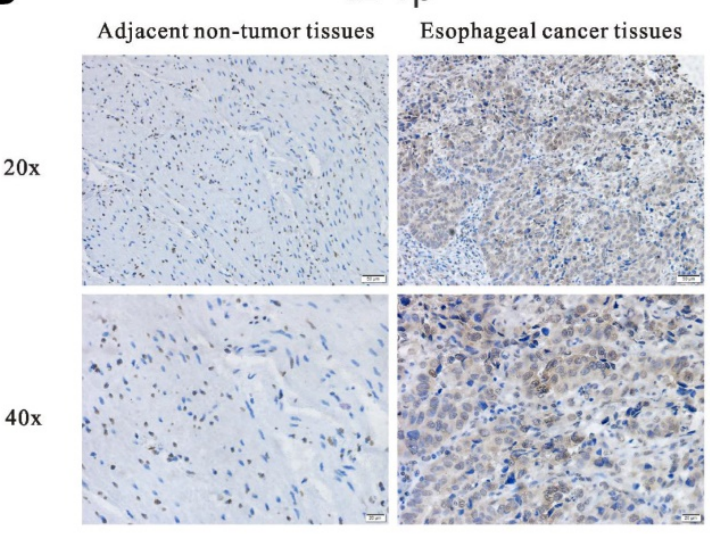

D

IL-1 $1 \beta$

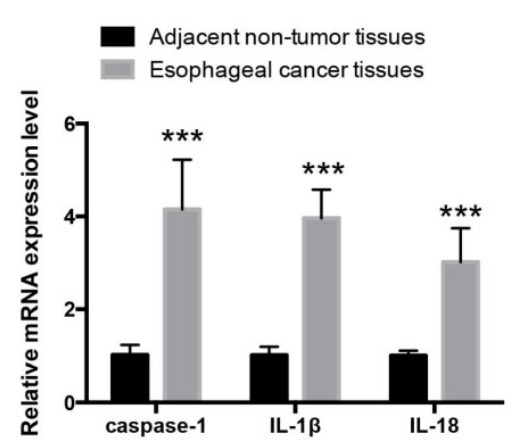

Adjacent non-tumor tissues

Esophageal cancer tissues
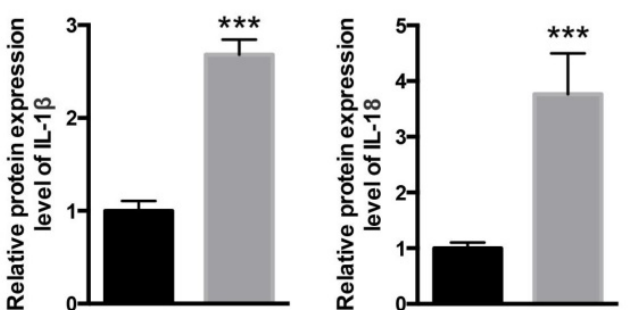

FIGURE 6. Pyroptosis may participate in esophageal cancer progression. Isolation of esophageal cancer tissues and adjacent non-tumor tissues and extraction of the whole nucleic acid and protein. Immunohistochemical staining of caspase-1 (A), IL-1 $\beta$ (B) and IL-18 (C) in adjacent normal tissues and esophageal cancer tissues. D. The mRNA expression levels of caspase-1, IL-1 $\beta$ and IL-18 were detected by real-time PCR. E. The protein expression levels of caspase-1, IL-1 $\beta$ and IL-18 were detected by western blotting. The data are representative of five independent experiments and represent the means \pm SD. $* * *, p<0.001$.

Therefore, esophagitis is being largely viewed as one of the complications of GERD [43-50]. To understand the mechanism of the occurrence of esophagitis could help us prevent diseases effectively. Several studies showed that alcohol consumption was associated with an increased risk of reflux esophagitis $[9,51,52]$. Our study clarified that alcohol could induce esophagitis, agreeing with previous studies that alcohol consumption tends to be associated with an increased risk of erosive esophagitis and Barrett's epithelium [53]. Furthermore, alcohol accumulation could activate caspase-1, IL-1 $\beta$ and IL-18, amplifying the inflammatory response to aggravate the progress of diseases through pyroptosis pathway. Pyroptosis is a caspase-1/caspase-11-dependent inflammatory form of cell death, which not only protects multicellular organisms from invasion by pathogenic bacteria and microbial infections but can also lead to sepsis and lethal septic shock if overactivated [54]. Caspase- 1 is an inflammatory caspase that is activated through the formation of inflammasome complexes in response to both pathogen-derived and endogenous mediators. The most well-known function of active caspase- 1 is to cleave the proforms of inflammatory cytokines IL-1 $\beta$ and -18 into their active forms in response to inflammatory stimuli in immune cells [55, 56]. A recent study suggests that caspase- 1 has multiple functions, in addition to this cytokine maturation role, and it is at the center of many cell responses to stress and inflammation [57]. AC-YVAD-CMK, an inhibitor of caspase-1, could effectively inhibit caspase-1 expression, and inhibit 
pyroptosis in many diseases [58-63]. Previous studies showed that $\mathrm{H}$. pylori-associated inflammation plays a role in the prevention of the development of erosive esophagitis[64, 65]. Our study, for the first time, identified that alcohol accumulation could cause inflammatory response and induce pyroptosis. However, Ac-YVAD-CMK, as the inhibitor of caspase-1, could significantly inhibit the expression of inflammatory cytokine IL-1 $\beta$ and IL-18 both in vivo and in vitro, thereby relieving the progress of esophagitis. Previous studies have shown that the caspase-1 inhibitor AC-YVAD-CMK could attenuate acute gastric injury, reducing acute lung injury and protecting the kidney from acute ischemic lesions [66-68]. However, no study has reported on the effect of AC-YVAD-CMK on esophagitis. Our study suggests that Ac-YVAD-CMK may be an effective reagent to suppress the development of esophagitis and defend against mucosal damage. Alcohol accumulation not only induces esophagitis but is associated with the pathogenesis of esophageal cancer [69-74]. Therefore, we detected the expression of caspase-1, IL-1 $\beta$ and IL-18 to identify whether pyroptosis plays roles during the progression of esophageal cancer. As expected, pyroptosis also participates in esophageal cancer. Our study showed that the caspase- 1 inhibitor AC-YVAD-CMK may be an effective agent to treat esophagitis caused by alcohol and may function in the treatment of esophageal cancer.

\section{Experimental Procedures}

HEEC cell lines and culture conditions-Human esophageal endothelial cells were purchased from the iCell (Shanghai, China). The cell lines were cultured in DMEM medium supplemented with $10 \%$ fetal bovine serum (FBS; Gibco, Australia), $100 \mathrm{U} / \mathrm{mL}$ penicillin, and $50 \mu \mathrm{g} / \mathrm{mL}$ streptomycin and were kept in incubators with humidified atmospheres of $5 \% \mathrm{CO}_{2}$ and $95 \%$ air at $37^{\circ} \mathrm{C}$.

$q R T-P C R$ - Total RNA was extracted from EC tissue samples and adjacent non-tumor tissue samples using TRIzol reagent (Invitrogen, Carlsbad, CA, USA), according to the manufacturer's instructions. Approximately $1 \mu \mathrm{g}$ of RNA was used to synthesize cDNA. The expression levels of caspase-1, IL-18 and IL-1 $\beta$ were determined by qPCR (ABI 7500fast system, Applied Biosystems, CA, USA), using GAPDH as the endogenous control. qRT-PCR results were expressed relative to the ratio of caspase-1, IL-18 or IL-1 $\beta$ and GAPDH expression. The forward and reverse primer sequences for caspase- 1 are 5' TTTCCGCAAGGTTC GATTTTCA 3' and 5' GGCATCTGCGCTCTACCATC $3^{\prime}$, respectively. For IL-1 $\beta$ : forward 5' ATGATGGCTT ATTACAGTGGCAA 3', reverse 5' GTCGGAGATTC
GTAGCTGGA 3'; Il-18: Forward 5' GCAAAGCTT ATGACCATGAGACACAACTG 3', Reverse 5' GCGAATTCGTCGACTTAACCCTGCTGCTGTGGA CT 3'.

MTT assay-Cells were seeded into 96-well plates and were treated as previously described. After treatment, $10 \mu \mathrm{l}$ of MTT reagent $(0.5 \mathrm{mg} / \mathrm{ml})$ was added to each well and was incubated for $4 \mathrm{~h}$ at $37^{\circ} \mathrm{C}$. The formazine granules in the wells were dissolved with $150 \mu \mathrm{l}$ of dimethyl sulfoxide (DMSO), and the absorbance at $570 \mathrm{~nm}$ was measured using a microplate reader.

HE staining-Hematoxylin and eosin (HE) staining was used to observe the histological changes. Tissues were fixed in $4 \%$ paraformaldehyde and embedded in paraffin. The samples were cut into 5 - $\mu$ m-thick sections and stained with HE.

Immunofluorescence-The cells were washed three times with PBS and then were fixed with $4 \%$ paraformaldehyde at room temperature for $15 \mathrm{~min}$. The cells were washed three times with PBS again and blocked with PBS containing 1\% bovine serum albumin (BSA) for $30 \mathrm{~min}$ at room temperature. Cells were then incubated overnight at $4^{\circ} \mathrm{C}$ with primary antibody diluted in blocking buffer and with secondary antibody for $1 \mathrm{~h}$ at room temperature. Caspase-1 was detected via a monoclonal anticaspase1 antibody (Cell Signaling; 2225) at a dilution of 1:200 and a horseradish peroxidase-conjugated goat anti-rabbit IgG antibody at a dilution of 1:1000. The cells were then washed three times with PBS. Images were taken using an Axiovert 200 fluorescence microscope.

Immunohistochemical staining - For immunohistochemical analysis, frozen esophageal section specimens were fixed with $4 \%$ buffered paraformaldehyde embedded in paraffin. Specimens were dehydrated by an ascending series of ethanol and cleared with xylene. All sections were immunostained with primary antibodies against caspase-1, IL-18 and IL-1 $\beta$ at $4^{\circ} \mathrm{C}$ overnight. After incubation with secondary antibodies, the sections were stained with diaminobenzidine.

Enzyme-linked immunosorbent assay-Culture medium was collected for the measurement of IL-1 $\beta$ and IL-18 using an ELISA kit (uscn-SEA064R and uscn-SEA563Ra) according to the manufacturer's instructions [37].

Western blot analysis - Total protein was extracted from the cells using RIPA buffer containing phenylmethanesulfonylfluoride (PMSF). Total protein concentrations were measured using a BCA Protein Assay Kit (Pierce, Rockford, IL). Thirty micrograms of the protein lysate was subjected to sodium dodecyl sulfate-polyacrylamide gel electrophoresis (SDS- 
PAGE) and were transferred onto PVDF membranes. The PVDF membranes were blocked with 5\% BSA in $0.05 \%$ Tween 20-TBS for 1 hour and were incubated with the corresponding primary antibody diluted in blocking buffer overnight at $4^{\circ} \mathrm{C}$. The dilutions for primary antibodies were as follows: anti-caspase-1 (1:1000, Cell Signaling; 2225), anti-IL-1 $\beta$ and anti-IL-18 (1:400; Santa Cruz Biotech). After extensive washing with TBST, anti-rabbit IgG-HRP secondary antibody (1:5,000; Santa Cruz Biotech) was added. Signals were determined using SuperSignal West Pico chemiluminescent substrate (Thermo, USA).

Terminal deoxynucleotidyl transferase (TdT)mediated-digoxigenin-11-dUTP nick end labeling (TUNEL)-TUNEL was used to detect DNA fragmentation in HEECs treated in different conditions according to the instructions of the TUNEL kit.

Statistical analyses - All statistical analyses were performed using SPSS 17.0 software. Student's $t$-test or one-way ANOVA was conducted for normally distributed data. All data were expressed as means \pm SD. Statistical significance was set at $P<0.05$.

Human studies - Human samples were obtained from 30 esophageal cancer patients in Department of Thoracic Surgery, Harbin Medical University Cancer Hospital. All these patient had at least 20 years' drinking history and 6 paired tissues were chosen randomly for further studies. All individuals provided informed consent for donation on approved institutional protocols.

Mouse studies-Fifteen male C57BL/6 mice weighting $20 \mathrm{~g}$ were obtained from the Experimental Animal Center of Harbin Medical University. These mice were divided into three groups with five mice in each group and were fed separately.As mice prowl at night looking for food, the experiments were conducted from $7 \mathrm{pm}$ to $7 \mathrm{am}$ of the next day. Control group were fed with physiological saline and food. Ethanol group and ethanol+inhibitor group were fed with $1 \%$ ethanol and same food. Meanwhile, mice in ethanol+inhibitor group were administered with caspase1 inhibitor. We recorded the weight changes and drinking volume of each mice. In control group the drinking volume was about $3 \mathrm{ml}$ everyday, and the other two groups were $1.5 \mathrm{ml}$. At $3 \mathrm{~d}$ after treatment, the mice were anesthetized and euthanized by cervical dislocation. The esophagus tissues were harvested and rinsed with saline and used for the following detection.

Ethics statement - The study was approved by the ethics committee of Harbin Medical University, and all experimental procedures were approved by the Animal Care and Use Committee of Harbin Medical University. Our study was performed in accordance with the recommendations of the Guide for the Care and Use of Laboratory Animals, published by the US National Institutes of Health (NIH Publication no. 85-23, revised 1996).

\section{Abbreviations}

GERD, Gastroesophageal reflux disease; HEECs, Human esophageal endothelial cells.

\section{Acknowledgments}

This work was supported by Hai Yan Foundation, Health Department of Heilongjiang Province of China, Harbin Bureau Science and Technology, Development Center for Medical Science and Technology (Grant No. JJMS2014-04, JJQN2016-05, 2013086, 2015RAQYJ101, W2012RQ29).

\section{Author contributions}

Fengjiao Wang, Gang $\mathrm{Li}$ and Jianqun Ma conceived and coordinated the study and wrote the paper. Jinfeng Ning, Lantao Chen, Hai $\mathrm{Xu}$, Xianglong Kong and Jianlong $\mathrm{Bu}$ performed and analyzed the experiments shown in Figure 1-6. Weiwei Zhao, Zhengtian Li, Xiuyun Wang, Xiaoguang Li provided technical assistance and contributed to the preparation of the figures. All authors reviewed the results and approved the final version of the manuscript.

\section{Competing Interests}

The authors have declared that no competing interest exists.

\section{References}

1. Dent J, El-Serag HB, Wallander MA, et al. Epidemiology of gastro-oesophageal reflux disease: a systematic review. Gut. 2005; 54(5):710-7.

2. Mitchell DR, Derakhshan MH, Robertson EV, et al. The Role of the Acid Pocket in Gastroesophageal Reflux Disease. J Clin Gastroenterol. 2016; 50(2):111-9.

3. Altomare A, Guarino MP, Cocca S, et al. Gastroesophageal reflux disease: Update on inflammation and symptom perception. World J Gastroenterol. 2013; 19(39):6523-8.

4. Serra Pueyo J. Update on gastroesophageal reflux disease. Gastroenterol Hepatol. 2014; 37(2):73-82.

5. Lagergren J, Bergström R, Lindgren A, et al. Symptomatic gastroesophageal reflux as a risk factor for esophageal adenocarcinoma. N Engl J Med. 1999; 340(11):825-31.

6. Akiyama $\mathrm{T}$, Inamori $\mathrm{M}$, Iida $\mathrm{H}$, et al. Shape of Barrett's epithelium is associated with prevalence of erosive esophagitis. World J Gastroenterol. 2010; 16(4):484-9.

7. Spechler SJ, Souza RF. Barrett's esophagus. N Engl J Med. 2014; 371(9):836-45.

8. Huo X, Zhang X, Yu C, et al. In oesophageal squamous cells exposed to acidic bile salt medium, omeprazole inhibits IL-8 expression through effects on nuclear factor-kB and activator protein-1. Gut. 2014; 63(7):1042-52.

9. Akiyama $\mathrm{T}$, Inamori $\mathrm{M}$, lida $\mathrm{H}$, et al. Alcohol consumption is associated with an increased risk of erosive esophagitis and Barrett's epithelium in Japanese men. BMC Gastroenterol. 2008;11;8:58.

10. Kaufman SE, Kaye MD. Induction of gastro-esophageal reflux by alcohol. Gut. 1978; 19(4):336-8. 
11. Keshavarzian A, Polepalle C, et al. Esophageal motor disorder in alcoholics: Result of alcoholism or withdrawal? Alcohol Clin Exp Res. 1990; 14(4):561-7.

12. Mincis M, Chebli JM, Khouri ST, et al. Ethanol and the gastrointestinal tract. Arq Gastroenterol. 1995; 32(3):131-9.

13. Kelly M, Hwang JM, Kubes P. Modulating leukocyte recruitment in inflammation. J Allergy Clin Immunol. 2007; 120(1):3-10.

14. Grad S, Abenavoli L, Dumitrascu DL. The Effect of Alcohol on Gastrointestinal Motility. Rev Recent Clin Trials. 2016; 11(3):191-5.

15. Toh Y, Oki E, Ohgaki K, et al. Alcohol drinking, cigarette smoking, and the development of squamous cell carcinoma of the esophagus: molecular mechanisms of carcinogenesis. Int J Clin Oncol. 2010; 15(2):135-44.

16. Haas SL, Ye W, Löhr JM. Alcohol consumption and digestive tract cancer. Curr Opin Clin Nutr Metab Care. 2012; 15(5):457-67.

17. Teyssen S, Singer MV. Alcohol-related diseases of the oesophagus and stomach. Best Pract Res Clin Gastroenterol. 2003; 17(4):557-73.

18. Rocco A, Compare D, Angrisani D, et al. Alcoholic disease: liver and beyond. World J Gastroenterol. 2014; 20(40):14652-9.

19. Siegmund SV, Singer MV. Effects of alcohol on the upper gastrointestinal tract and the pancreas--an up-to-date overview. Z Gastroenterol. 2005; 43(8):723-36.

20. He Y, Amer A O. Microbial modulation of host apoptosis and pyroptosis. Frontiers in Cellular \& Infection Microbiology. 2014; $4(4): 83-83$.

21. Hu P, Huang F, Niu J, et al. TLR-4 involvement in pyroptosis of mice with pulmonary inflammation infected by Actinobacillus pleuropneumoniae. Acta Microbiologica Sinica. 2015; 55(5):650-6.

22. Bergsbaken T, Fink S L, Cookson B T. Pyroptosis: host cell death and inflammation. Nature Reviews Microbiology. 2009; 7(7):99-109.

23. Boudreau N, Sympson CJ, Werb Z, et al. Suppression of ICE and apoptosis in mammary epithelial cells by extracellular matrix. Science. 1995; 267(5199):891-3.

24. Sollberger G, Strittmatter GE, Garstkiewicz M, et al. Caspase-1: the inflammasome and beyond. Innate Immun. 2014; 20(2):115-25.

25. Martinon F, Tschopp J. Inflammatory caspases: linking an intracellular innate immune system to autoinflammatory diseases. Cell. 2004; 117(5):561-74.

26. Martinon F, Tschopp J. Inflammatory caspases and inflammasomes: master switches of inflammation. Cell Death Differ. 2007; 14(1):10-22.

27. Delaleu N, Bickel M. Interleukin-1 $\beta$ and interleukin-18: regulation and activity in local inflammation. Periodontol. 2004; 35:42-52.

28. Ryan JG, Goldbach-Mansky R. The spectrum of autoinflammatory diseases: recent bench to bedside observations. Curr Opin Rheumatol. 2008; 20(1):66-75.

29. Satoh T, Otsuka A, Contassot E, et al. The inflammasome and IL-1 $\beta$ : implications for the treatment of inflammatory diseases. Immunotherapy. 2015; 7(3):243-54.

30. Liao Z, Xiao HT, Zhang Y, et al. IL-1 $\beta$ : a key modulator in asthmatic airway smooth muscle hyper-reactivity. Expert Rev Respir Med. 2015; 9(4):429-36.

31. Nakanishi $\mathrm{K}$, Yoshimoto $\mathrm{T}$, Tsutsui $\mathrm{H}$, et al. Interleukin-18 regulates both Th1 and Th2 responses. Annu. Rev. Immunol. 2001; 19:423-474

32. Martinon F, Burns K, Tschopp J. The inflammasome: a molecular platform triggering activation of inflammatory caspases and processing of proIL-beta. Mol Cell. 2002; 10(2):417-26.

33. Fink SL, Cookson BT. Caspase-1-dependent pore formation during pyroptosis leads to osmotic lysis of infected host macrophages. Cellular microbiology. 2006; 8:1812-1825.

34. Miao EA, Rajan JV, Aderem A. Caspase-1-induced pyroptotic cell death. Immunol Rev. 2011; 243(1):206-14.

35. Wallach D, Kang TB, Dillon CP, et al. Programmed necrosis in inflammation: Toward identification of the effector molecules. Science. 2016; 352(6281):aaf2154

36. Croker BA, Silke J, Gerlic M. Fight or flight: regulation of emergency hematopoiesis by pyroptosis and necroptosis. Curr Opin Hematol. 2015; 22(4):293-301.

37. Lee HS, Jeon SW. Barrett esophagus in Asia: same disease with different pattern. Clin Endosc. 2014; 47(1):15-22.

38. Howard PJ, Maher L, Pryde A, et al. Symptomatic gastro-oesophageal reflux, abnormal oesophageal acid exposure, and mucosal acid sensitivity are three separate, though related, aspects of gastro-oesophageal reflux disease. Gut. 1991; 32: 128-32.

39. Baldi F, Ferrarini F, Longanesi A, et al. Acid gastroesophageal reflux and symptom occurrence. Analysis of some factors influencing their association. Dig Dis Sci. 1989; 34(12):1890-3.

40. Weusten BL, Akkermans LM, VanBerge-Henegouwen GP, et al. Symptom, perception in gastroesophageal reflux disease is dependent on spatiotemporal reflux characteristics. Gastroenterology. 1995; 108: 1739-44.

41. Cicala M, Emerenziani S, Caviglia R, et al. Intra-oesophageal distribution and perception of acid reflux in patients with non-erosive gastro-esophageal reflux disease. Aliment Pharmacol Ther. 2003; 18: 605-13.

42. Bredenoord AJ, Weusten BL, Curvers WL, et al. Determinants of perception of heartburn and regurgitation. Gut. 2006; 55(3):313-8.

43. Dent J, El-Serag HB, Wallander MA, et al. Epidemiology of gastro-oesophageal reflux disease: a systemic review. Gut. 2005; 54(5):710-7.

44. Jones RH, Hungin AP, Philips J, et al. Gastro-oesophageal reflux disease in primary care in Europe: clinical presentation and endoscopic findings. Eur J Gen Pract. 1995; 1: 149-54.

45. Carlsson R, Dent J, Watts R, et al. Gastro-oesophageal reflux disease in primary care: an international study of different treatment strategies with omeprazole. International GORD Study Group. Eur J Gastroenterol Hepatol. 1998; 10: 119-24.

46. Okamoto K, Iwakiri R, Mori M, et al. Clinical symptoms in endoscopic reflux esophagitis: evaluation in 8031 adult subjects. Dig Dis Sci. 2003; 48: 2237-41

47. Quigley EM, DiBaise JK. Non-erosive reflux disease: the real problem in gastro-oesophageal reflux disease. Dig Liver Dis. 2001; 33: 523-7.

48. Martinez SD, Malagon IB, Garewal HS, et al. Non-erosive reflux disease (NERD) - acid reflux and symptom patterns. Aliment Pharmacol Ther. 2003; 17: 537-45.

49. DeVault KR, Castell DO. Updated guidelines for the diagnosis and treatment of gastroesophageal reflux disease. Am J Gastroenterol. 2005; 100: 190-200.

50. Fujiwara $\mathrm{Y}$, Higuchi $\mathrm{K}$, Shiba $\mathrm{M}$, et al. Differences in clinical characteristics between patients with endoscopy-negative reflux disease and erosive esophagitis in Japan. Am J Gastroenterol. 2005; 100: 754-8.

51. Minatsuki C, Yamamichi N, Shimamoto T, et al. Background factors of reflux esophagitis and non-erosive reflux disease: a cross-sectional study of 10,837 subjects in Japan. PLoS One. 2013; 8(7):e69891.

52. Gunji T, Sato H, Iijima K, et al. Risk factors for erosive esophagitis: a cross-sectional study of a large number of Japanese males. J Gastroenterol. 2011; 46(4):448-55.

53. Chung TH, Lee J, Kim MC. Impact of night-shift work on the prevalence of erosive esophagitis in shipyard male workers. Int Arch Occup Environ Health. 2016; 89(6):961-6.

54. Aglietti RA, Dueber EC. Recent Insights into the Molecular Mechanisms Underlying Pyroptosis and Gasdermin Family Functions. Trends Immunol. 2017; 38(4):261-271.

55. Sun $\mathrm{Q}$, Scott M. Caspase-1 as a multifunctional inflammatory mediator: noncytokine maturation roles. J Leukoc Biol. 2016; 100(5):961-967.

56. Patel MN, Carroll RG, Galván-Peña S, et al. Inflammasome Priming in Sterile Inflammatory Disease. Trends Mol Med. 2017; 23(2):165-180.

57. Sun Q, Scott M. Caspase-1 as a multifunctional inflammatory mediator: noncytokine maturation roles. J Leukoc Biol. 2016; 100(5):961-967.

58. Gemma C, Fister M, Hudson C, et al. Improvement of memory for context by inhibition of caspase-1 in aged rats. Eur J Neurosci. 2005; 22(7):1751-6.

59. Liang DY, Li X, Li WW, et al. Caspase-1 modulates incisional sensitization and inflammation. Anesthesiology. 2010; 113(4):945-56.

60. Jin $\mathrm{Y}, \mathrm{Li} \mathrm{H}, \mathrm{Xie} \mathrm{G}$, et al. Sevoflurane combined with ATP activates caspase-1 and triggers caspase-1-dependent pyroptosis in murine J774 macrophages. Inflammation. 2013; 36(2):330-6.

61. Wu B, Ma Q, Khatibi N, et al. Ac-YVAD-CMK Decreases Blood-Brain Barrier Degradation by Inhibiting Caspase-1 Activation of Interleukin-1 $\beta$ in Intracerebral Hemorrhage Mouse Model. Transl Stroke Res. 2010; 1(1):57-64.

62. Lipinska $\mathrm{K}$, Malone KE, Moerland $\mathrm{M}$, et al. Applying caspase-1 inhibitors for inflammasome assays in human whole blood. J Immunol Methods. 2014; 411:66-9

63. Mathiak G, Grass G, Herzmann $T$, et al. Caspase-1-inhibitor ac-YVAD-cmk reduces LPS-lethality in rats without affecting haematology or cytokine responses. Br J Pharmacol. 2000; 131(3):383-6.

64. Unal S, Karakan T, Dogan I, et al. The influence of Helicobacter pylori infection on the prevalence of endoscopic erosive esophagitis. Helicobacter. 2006; 11: 556-61.

65. Nordenstedt H, Nilsson M, Johnsen R, et al. Helicobacter pylori infection and gastroesophageal reflux in a population-based study (The HUNT Study). Helicobacter. 2007; 12(1):16-22.

66. Zhang F, Wang L, Wang JJ, et al. The caspase-1 inhibitor AC-YVAD-CMK attenuates acute gastric injury in mice: involvement of silencing NLRP3 inflammasome activities. Sci Rep. 2016; 6:24166. 
67. Wu DD, Pan PH, Liu B, et al. Inhibition of Alveolar Macrophage Pyroptosis Reduces Lipopolysaccharide-induced Acute Lung Injury in Mice. Chin Med J (Engl). 2015; 128(19):2638-45.

68. Liu HF, Liang D, Wang LM, et al. Effects of specific interleukin-1beta-converting enzyme inhibitor on ischemic acute renal failure in murine models. Acta Pharmacol Sin. 2005; 26(11):1345-51.

69. Nieminen MT, Novak-Frazer L, Collins R, et al. Alcohol and acetaldehyde in African fermented milk mursik--a possible etiologic factor for high incidence of esophageal cancer in western Kenya. Cancer Epidemiol Biomarkers Prev. 2013; 22(1):69-75.

70. Jelski W, Laniewska-Dunaj M, Niklinski J, et al. The alcohol dehydrogenase isoenzyme (ADH IV) as a candidate tumour marker of esophageal cancer. Acta Biochim Pol. 2013; 60(3):489-93.

71. Palladino-Davis AG, Mendez BM, Fisichella PM, et al. Dietary habits and esophageal cancer. Dis Esophagus. 2015; 28(1):59-67.

72. Roerecke M, Shield KD, Higuchi S, et al. Estimates of alcohol-related oesophageal cancer burden in Japan: systematic review and meta-analyses. Bull World Health Organ. 2015; 93(5):329-338C.

73. Oze I, Matsuo K, Wakai K, et al. Alcohol drinking and esophageal cancer risk: an evaluation based on a systematic review of epidemiologic evidence among the Japanese population. Jpn J Clin Oncol. 2011; 41(5):677-92

74. Ding JH, Li SP, Cao HX, et al. Alcohol dehydrogenase-2 and aldehyde dehydrogenase-2 genotypes, alcohol drinking and the risk for esophageal cancer in a Chinese population. J Hum Genet. 2010; 55(2):97-102. 